Check for updates

Cite this: RSC Adv., 2017, 7, 37755

Received 6th May 2017

Accepted 6th July 2017

DOI: $10.1039 / c 7 r a 05118 b$

rsc.li/rsc-advances

\section{Adsorption characteristics of Cytochrome c/DNA complex Langmuir molecular assemblies at the air- water interface: a surface area-normalized isotherm study}

\author{
Pabitra Kumar Paul, (DD ab Dock-Chil Che, ${ }^{a}$ Kishimoto Hiroyuki, ${ }^{a}$ Kento Araki ${ }^{a}$ \\ and Takuya Matsumoto*a
}

\begin{abstract}
We present the formation of a complex molecular network consisting of highly water soluble $\lambda$-DNA and a redox protein, Cytochrome $c$ (Cyt $c$ ), at the air-water interface by Langmuir-Blodgett technique. Time dependent adsorption of these materials and the formation of a Cyt $c / \lambda$-DNA complex interfacial layer in the Langmuir trough were systematically studied by recording surface pressure versus trough area isotherms after various subphase incubation times. We have succeeded in separating two effects: one is the phase change of the monolayers and the other is the number of molecules participating in the monolayer formation with the change in incubation time using a normalized isotherm in units of critical surface area. The interaction in the complex monolayer was found to be same as that of single component $\lambda$-DNA during $3 \mathrm{~h}$ of subphase incubation. However, it was also similar to that of a single component Cyt $\mathrm{c}$ monolayer after $5 \mathrm{~h}$ incubation. The morphology and electrical polarizations of these interfacial films deposited onto a mica substrate were analyzed by Atomic Force Microscopy and electrostatic force microscopy respectively. It was found that Cyt $c$ and $\lambda$-DNA immediately form the complex network at the air-water interface, and change to rod-like large aggregates with prolonged incubation.
\end{abstract}

\section{Introduction}

Interactions of deoxyribonucleic acid (DNA) with water soluble proteins have attracted great attention in recent years for fundamental importance due to their involvements in some specific and non-specific interactions. ${ }^{1}$ The specific type of interaction can be manifested as the intercalation of amino acid residues between the DNA base pairs or protein can bind to the DNA secondary structures. ${ }^{2}$ But the non-specific binding arises due to electrostatic interactions between cationic parts of the protein with the anionic phosphate parts of DNA and this complexation is comparatively less stable as it excludes hydrophobic interactions. Also the strength of the DNA-protein assembly may depend upon the strength of the surrounding ionic environment. In recent times, DNA based nanoarchitechtures have attracted widespread interest because of their significant role in developing nanoscaled scaffolds, frameworks and wires..$^{3-6}$ On the other hand, electron transfer between proteins plays a vital role both in photosynthesis which

\footnotetext{
${ }^{a}$ Department of Chemistry, Graduate School of Science, Osaka University, 1-1 Machikaneyama-Cho, Toyonaka, Osaka,560-0043, Japan.E-mail: matsumoto-t@ chem.sci.osaka-u.ac.jp

${ }^{b}$ Department of Physics, Jadavpur University, Jadavpur, Kolkata-700 032, India
}

harvests light energy as well as in metabolism that oxidizes fuel. This long range energy transfer may be attributed to the wellcontrolled interfacial associations of proteins. ${ }^{7}$

From the biotechnological point of view, the study on the interactions of DNA with protein is extremely important because of their possible applications namely, molecular recognition, enzyme immobilization etc. $^{8-10}$ Some reports suggest that 3D DNA arrangements (also known as DNA origami) ${ }^{11}$ are capable of assembling proteins while preserving their functionalities. ${ }^{12-14}$ However, it is of emerging interest to proceed first for more controllable interfacial associations of electroactive protein molecules within DNA chains both for fundamental as well as technological applications especially in bio-molecular electronics such as advanced bio-sensing, artificial neuron-based information processing devices etc. utilizing their nanoscale electron transfer processes. ${ }^{15-17}$ Also the rate and nature of electron transfer process may be realized by manipulating the organizations of their complex molecular assemblies at a nanometer scale. DNA has already been used to prepare some supramolecular network as the building block for assembling of redox protein namely, Cytochrome $c$ (Cyt $c$ ) into multilayers $^{18}$ via layer-layer-layer self-assembly method. However, there was no such efficient and precise control of molecular orientation as well as the spatial distribution of 
redox-active centres of protein in the complex architectures. Although this ordered DNA network has some potential for their role in direct electron transport through redox proteins ${ }^{\mathbf{1 8}}$ but their industrial applications may be limited due to comparably less control over the molecular engineering of DNA assemblies onto solid substrate. Therefore, more experimental sophistication may be necessary which may require additional cost for large-scale of such nanofabrication. Recently, Kawai et al. ${ }^{\mathbf{1 9 , 2 0}}$ also reported the molecular network arrangements of DNA organized onto solid substrate (mica or HOPG).

Langmuir-Blodgett (LB) method has long been acknowledged as an excellent approach to study the surface activity of protein and nucleic acids $\mathbf{s}^{\mathbf{2 1 , 2 2}}$ at air-water interface and to prepare their stable ultrathin films onto solid substrates. It is well known that protein molecules have high tendency to accumulate at their phase boundary at the surfaces. ${ }^{23}$ Many biological processes such as cell adhesion, blood clotting and so forth are basically the manifestations of the surface characteristics of proteins at the interfaces. The highly hydrophilic DNA molecule has been adsorbed onto oppositely charged surfactant monolayer at air-water interface and the resultant complex assemblies is easily immobilized onto solid substrate via LB method. ${ }^{22,24}$ In a recent work it is observed that only DNA can also form a well-ordered molecular network at air-water interface by means of LB technique. ${ }^{25}$ However, the reason for this unusual phase behaviour of DNA molecules at air-water interface is not yet clearly explored. Additionally this surface activity of DNA can provide a new dimension for their interactions with water soluble protein to form well-ordered DNA-protein complex molecular architectures at air-liquid interface. Therefore systematic study is of prior importance to probe the interactions involved in the organizations of such complex molecular assemblies prepared via LB technique which may influence the charge transfer reactions at their surfaces for suitable application.

In the present work, we basically address the formations of ordered complex molecular architecture composed of water soluble protein namely Cyt $c$ and nucleic acid $\lambda$-DNA at airwater interface and also successful transfer of this complex molecular assemblies onto solid substrate. Cyt $c$ is a small $\alpha$ helical heme protein with redox center and functions as an electron transporter in mitochondrial respirations. ${ }^{26}$ Isoelectric point (pI) of Cyt $c$ is 10.02-10.5 and the abundant charged lysine residues around the heme edge $\mathrm{e}^{27}$ is responsible for rapid interprotein electron transfer due to electrostatic steering. ${ }^{28} \mathrm{Cyt}$ $c$ at neutral $\mathrm{pH}$ has a net positively charge causing its stickiness and this also facilitates some strong and unspecific interactions. ${ }^{29}$ It can act as surfactant in reverse micellar solution. ${ }^{30,31}$ Research suggests that Cyt $c$ /DNA multilayered systems exhibit highest accumulations of redox active materials. ${ }^{32}$ However, for specific device or applications, it is very crucial to tailor the properties by changing the orientation of DNA/protein complex assemblies onto solid substrate. LB technique, in this regard, is an elegant choice because of its ability to manipulate molecular orientation and prepare ultrathin films with highest materials transfer ratio. ${ }^{33}$ So, this paper demonstrates the unusual surface activities of $\lambda$-DNA, Cyt $c$ and Cyt $c / \lambda$-DNA complex molecular assemblies at air-water interface by means of LB technique. Surface pressure $(\pi)$ versus area $(A)$ isotherms studies revealed the formation of their monolayer at air-water interface after allowing various subphase incubation times. The approach of normalized isotherms at a particular surface pressure and the critical surface area are introduced to probe their molecular level interactions during interfacial adsorption of Cyt $c$ and $\lambda$ DNA. Atomic Force Microscopy (AFM) in tapping mode has been utilized to understand the surface morphology of their LB films deposited onto solid substrate. Electrostatic Force Microscopic (EFM) study in frequency shift non-contact mode of LB film was also used to probe ionic interaction between $\lambda$-DNA and Cyt $c$ in the complex LB interfacial layer.

\section{Experimental}

\subsection{Materials used}

Cytochrome $c$ (Cyt $c$ ) from horse heart and $\lambda$-DNA were purchased from Sigma-Aldrich Chemical Company, USA and Takara Bio Inc., Japan respectively and used without further purification. Concentration of $\lambda$-DNA aqueous solution as purchased was $0.36 \mathrm{~g} \mathrm{l}^{-1}$. Mica substrates for depositing LB films were purchased from The Nilaco Corporation, Japan and were cut into $10 \mathrm{~mm} \times 10 \mathrm{~mm}$ pieces. Triple distilled deionised water (resistivity $18 \mathrm{M} \Omega \mathrm{cm}$ ) was prepared by Milipore Milli-Q water purification system (Model Number: DIRECT-Q 3 UV).

\subsection{Preparation of Langmuir monolayers and LB films}

The surface pressure-molecular area $(\pi-A)$ isotherms were prepared with a rectangular shaped Teflon coated trough fitted with two movable barriers attached to a fully computer controlled commercial LB film deposition instrument (622D1, NIMA, UK and Small, KSV NIMA, Finland). The surface pressure was recorded with the help of a Wilhelmy plate one end of which is attached to a pressure sensor and other end is partially immersed onto the aqueous subphase of Langmuir trough. The trough was cleaned by potassium permanganate solution for at least 10 hours followed by rigorous cleaning by ultrapure water. In order to remove any organic contaminants, acetone was used to clean finally the Langmuir trough before filling it with the subphase (i.e. triple distilled deionized water).

To obtain the $\pi-A$ isotherms of $\lambda$-DNA, Cyt $c$ or Cyt $c / \lambda$-DNA complex, $150 \mu \mathrm{l}$ of $\lambda$-DNA solution (concentration of $0.5 \mathrm{mg}$ $\mathrm{ml}^{-1}$ ), $25 \mu \mathrm{l}$ of Cyt $c$ solution (concentration of $10 \mathrm{mg} \mathrm{ml}^{-1}$ ), and the pre-mixed one of both these solutions were spread respectively by a microliter syringe onto the aqueous subphase in Langmuir trough keeping $\mathrm{pH}$ at 6.2 and temperature of $25^{\circ} \mathrm{C}$. After spreading the samples, the subphase surface was incubated for different hours prior to barrier compression for recording the pressure-area isotherms. The compression was performed by two movable barriers at a rate of $5 \mathrm{~mm} \mathrm{~min}^{-1}$. After attaining the desired surface pressure by compressing the surface with the barrier, the interfacial molecular layers were carefully transferred onto freshly cleaved mica substrate by horizontal dipping method and then the films were dried in air to prepare stable organized LB molecular films. 


\subsection{Characterizations of LB films by AFM and EFM}

The morphology of LB films of Cyt $c$ as well as Cyt $c / \lambda$-DNA complex were visualized and analysed by a commercial Scanning Probe Microscope (JSPM-4200, JEOL, Japan). The topographic images were obtained in tapping mode in air at ambient temperature and presented as raw data except little bit flattening to correct the background curvature of the substrate surface used for LB film deposition. The scanning tip used in the AFM measurement was a Si cantilever having a force constant of $25 \mathrm{~N} \mathrm{~m}^{-1}$ and resonant frequency of $300 \mathrm{kHz}$.

EFM images were obtained by frequency shift non-contact mode using phase-locked loop detector (OC4 station, NANONIS) and lock-in amplifier (1550, EG \& G, USA) at a pressure of 2 $\times 10^{-4}$ mbar with PtIr-coated cantilever having spring constant $42 \mathrm{Nm}^{-1}$ and resonant frequency of $330 \mathrm{kHz}$. The detail experimental design was illustrated in one of our previous work. ${ }^{34}$ The simultaneous topography and the EFM images were taken without any unexpected charge injection from the tip to the sample surface.

\section{Results and discussion}

\section{1. $\lambda$-DNA, Cyt $c$ and Cyt $c / \lambda$-DNA complex molecular assemblies at air-water interface}

The surface pressure $v s$. area $(\pi-A)$ isotherms of single component $\lambda$-DNA, single component Cyt $c$, and Cyt $c / \lambda$-DNA complex monolayers at air-water interface are shown in Fig. 1. In all the cases the subphase after sample spreading were incubated for

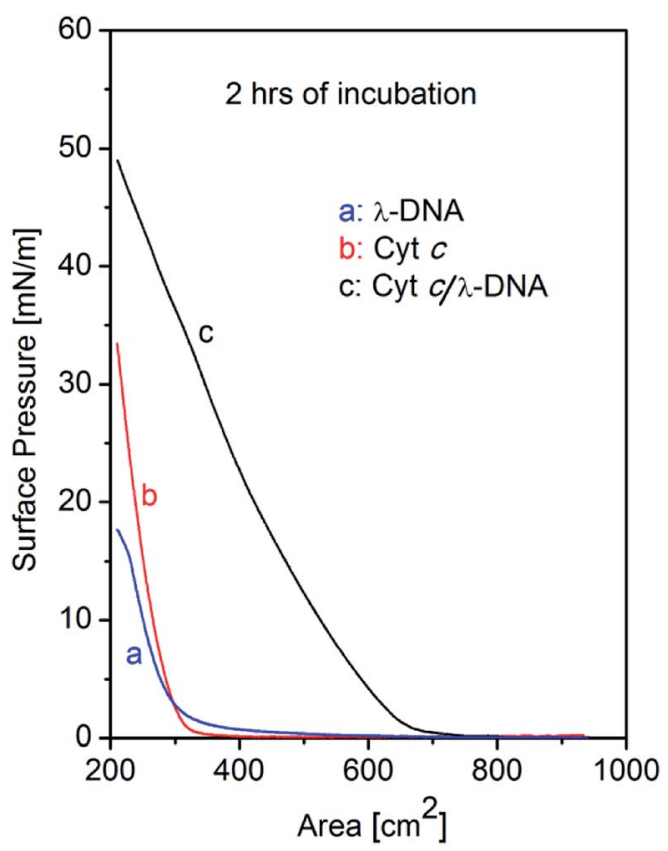

Fig. 1 Surface pressure $(\pi)$ versus trough area $(A)$ isotherms of $\lambda$-DNA (a), Cyt $c$ (b) and Cyt $c / \lambda$-DNA (c) complex monolayer at air-water interface. Barrier compression was done after $2 \mathrm{~h}$ of spreading the samples in the Langmuir trough. $\mathrm{pH}$ of the subphase was 6.2 in all the cases. The area of isotherm for Cyt $c / \lambda$-DNA (c) complex monolayer becomes higher as compared with single component Cyt $c$ or $\lambda$-DNA. two hours before barrier compression for recording the isotherms. It is well known that $\lambda$-DNA is well soluble in water. However, we observed that the surface pressure of $\lambda$-DNA increases upon compression by barrier at air-water interface. With the decrease in area of the subphase surface in Langmuir trough by movable barriers, the surface pressure rises very slowly from $c a .500 \mathrm{~cm}^{2}$ and the slope becomes steep gradually after reaching the surface area of $300 \mathrm{~cm}^{2}$. This tail and nonlinear behaviour of $\lambda$-DNA isotherm might attribute to the interactions between long-chained DNA molecules. In the present experimental condition, the double stranded DNA molecules should always remain in the bulk solution phase in the trough. Therefore, the unusual formation of DNA monolayer may be due to the self-association of DNA chains at air-water interface. Such rise in surface pressure of DNA with increase in subphase incubation time has been reported by Dai et al. ${ }^{25}$

The appearance of surface pressure was also observed for Cyt $c$ molecules which are well known as highly water soluble heme protein. However, the detailed behaviour of isotherms of the single component Cyt $c$ is different from that of $\lambda$-DNA. When the trough area becomes $320 \mathrm{~cm}^{2}$ during the compression of interface layer, there is a sharp rise in surface pressure of Cyt $c$ indicating the formation of condensed state of Cyt $c$ monolayer layer at air-water interface. The observed isotherm shows a small tail and linear increase in comparison with that of DNA. This arise of surface pressure corresponds to a critical surface concentration of the protein molecules and only above which it can engage in intermolecular cohesive interactions at air-water interface thereby resulting a greater reductions in surface tension. ${ }^{23}$ These results suggest that the interaction between Cyt $c$ molecules can be explained as simple hard spheres.

The molecular assemblies of single component Cyt $c$ at the surface pressure of $10 \mathrm{mN} \mathrm{m}^{-1}$ after $2 \mathrm{~h}$ of subphase incubation was transferred onto a mica substrate and the surface morphology of this LB monolayer film was observed by AFM as shown in Fig. 2. Cyt $c$ shows the close-packed particle-like images in the molecular layer with two different contrasts: the dark is $c a .1 \mathrm{~nm}$ for the majority and the bright is $c a .2 \mathrm{~nm}$ as shown in section profile of Fig. 2. The natural diameter of Cyt $c$ is known to be $\sim 3 \mathrm{~nm}$. However it is well known that the height of protein reduces on the surface by adsorption energy. The presence of two kinds of height might reflect the difference of adsorption energy due to the different orientation of Cyt $c$ molecules assembled on to the substrate. Another possible explanation is that the bright contrast indicates overlayer simply but this is unlikely because the bright molecules seem not to sit on the gap site of first layer molecules.

On the other hand, isotherm of Cyt $c / \lambda$-DNA complex molecular layer at air-water interface shows different behaviour when compared to the isotherms of single component protein or $\lambda$-DNA. The area of the isotherm at a particular surface pressure is higher as compared with the sum of the area of individual isotherms of $\lambda$-DNA and Cyt $c$ molecular layer. This clearly indicates the presence of complex formation between $\lambda$ DNA and Cyt $c$ at the air-water interface. It also implies that the bond formation between Cyt $c$ and $\lambda$-DNA proceeds effectively at air-water interface where less hydrophilic environment is 

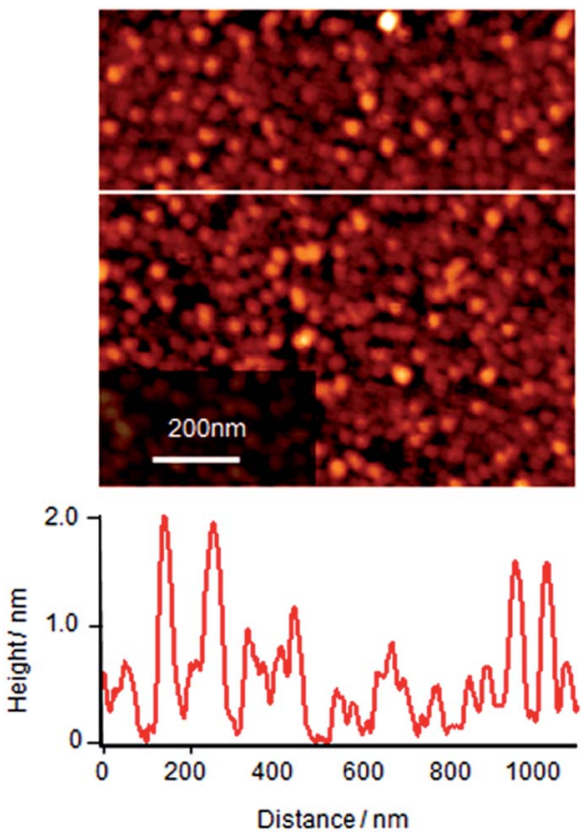

Fig. 2 AFM topographic image (tapping mode) of single component Cyt $c$ monolayer deposited onto mica at surface pressure of $10 \mathrm{mN}$ $\mathrm{m}^{-1}$. Barrier compression of the monolayer was done after $2 \mathrm{~h}$ of subphase incubation at air-water interface. Cyt $c$ molecules are observed as particles having two height components: one is the height of ca. $1 \mathrm{~nm}$, and the other is that of ca. $2 \mathrm{~nm}$.

favourable for less charged complex consisting of positive amino-residue of Cyt $c$ and negative phosphate backbone of DNA. Fig. 3 shows schematic illustration of the formation of Cyt

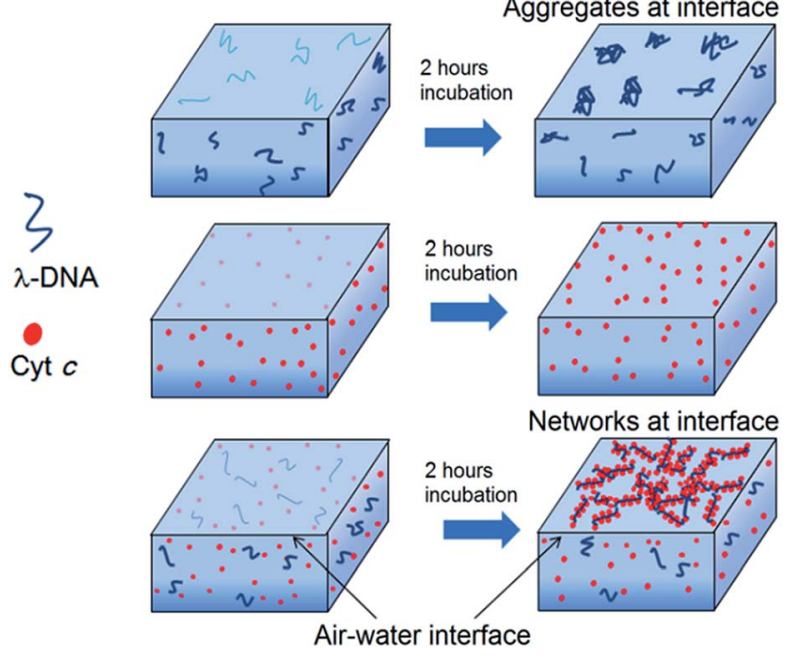

Fig. 3 Schematic illustration of the formation of Langmuir molecular layer of $\lambda$-DNA, single component of Cyt $c$ and Cyt $c / D N A$ complex at the air-water interface after $2 \mathrm{~h}$ incubation. The number of molecules participating at air-water interface increases during subphase incubation. The portion of the molecule at the air-water interface is determined by the equilibrium between the bulk water and the interface. Due to the formation of complex between Cyt $c$ and $\lambda$-DNA, the equilibrium moves to the interface and this is manifested as the observed high surface pressure at air-water interface. $c / \lambda$-DNA complex molecular assemblies at air-water interface. For both the separate isotherms of $\lambda$-DNA and Cyt $c$, the portion of the molecule at air-water interface is determined by the equilibrium between the bulk water and the interface. However, due to the formation of complex between Cyt $c$ and $\lambda$-DNA the equilibrium moves towards the interface and this is manifested as the observed high surface pressure in case of Cyt $c / \lambda$-DNA mixed monolayer at air-water interface.

The shape of Cyt $c / \lambda$-DNA complex isotherm shows rapid increase near critical area and small tail which is different from $\lambda$-DNA isotherm but almost same as that of Cyt $c$. This suggests that the motion of long-chained DNA in Cyt $c / \lambda$-DNA complex is considerably restrained even under weak surface pressure.

To understand the association and complex formation of Cyt $c$ and $\lambda$-DNA, molecular layer of Cyt $c / \lambda$-DNA complex (1 layer) was transferred onto mica substrate at surface pressure of 10 $\mathrm{mN} \mathrm{m}^{-1}$ after $2 \mathrm{~h}$ of subphase incubation (before running the isotherm). Fig. 4 shows the tapping mode AFM topographic image of Cyt $c / \lambda$-DNA molecular layer deposited onto mica. The section profile shows that the height of chain structure is $c a$. $3 \mathrm{~nm}$ which is clearly higher than $\lambda$-DNA diameter. It indicates that Cyt $c$ molecules are clearly bound to the $\lambda$-DNA chain. The image also reveals that Cyt $c$ plays a linker molecule between DNA chains. This result is consistent with the behaviour of surface pressure-area isotherm at the low pressure region as mentioned above.
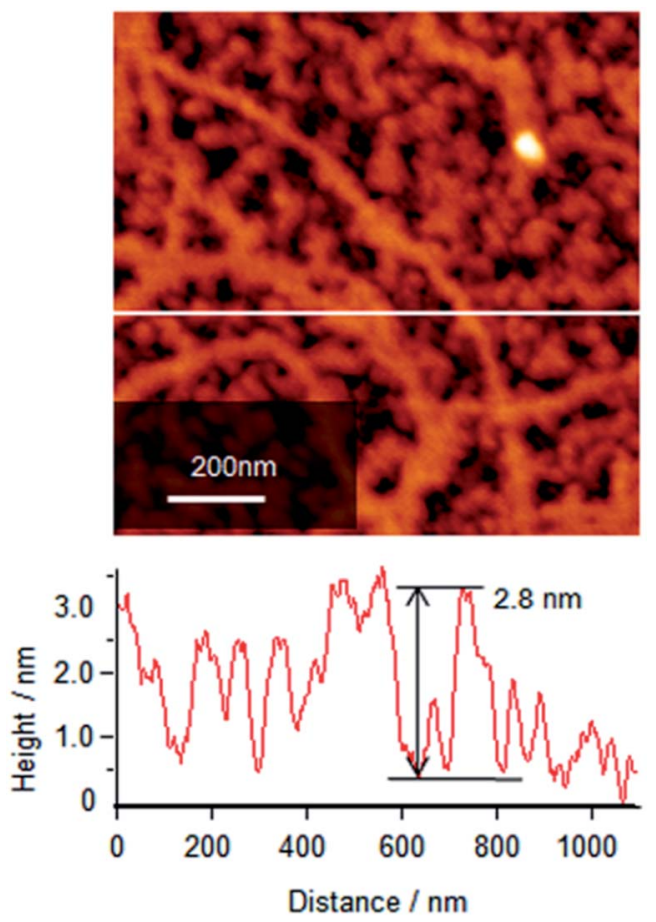

Fig. 4 AFM topographic image (tapping mode) of Cyt $c / \lambda$-DNA complex Langmuir monolayer transferred onto mica substrate at a surface pressure of $10 \mathrm{mN} \mathrm{m}^{-1}$ by horizontal dipping method. Barrier compression was done after $2 \mathrm{~h}$ of spreading Cyt $c / \lambda$-DNA mixed solution onto the subphase. Section profile of image shows the typical height of $2.8 \mathrm{~nm}$ which corresponds to the Cyt $c / \lambda$-DNA complex. 


\subsection{Effect of subphase incubation time: analysis by surface-} area-normalized isotherms

It is already observed in Fig. 1 that single component $\lambda$-DNA can form insoluble monolayer at air-water interface with incubation for long time. This result is consistent with a previous report for DNA self-assembly at air-water interface as was discussed earlier. ${ }^{25}$ However the behaviour of DNA molecules in the Langmuir trough has not been resolved and the behaviour of isotherm is being left unanalysed well. In general, the increase of surface pressure involves two effects: one is the phase change including the molecular arrangements, orientation, assembly, and/or, aggregations in monolayer, and the other is mere increase of the number of molecules participating the adsorption process at air-water interface. For the discrimination of these two effects, we have introduced the analysis of isotherms using normalization by surface area $(A)$ at a certain surface pressure $(\pi)$. In the surface-area-normalized isotherms, the slopes actually mean two-dimensional Young's modulus for the monolayer at that pressure and overall shapes reflect the behaviour of molecules at air-water interface excluding the effect of the number of molecules participating to the interface. In order to explore the effects of incubation time on the formation of monolayer at air-water interface, we have studied the surface pressure versus area isotherms of single component $\lambda$-DNA, single component Cyt $c$, and Cyt $c / \lambda$-DNA complex for different incubation time before running the isotherms as shown in Fig. 5-7, respectively.

The surface pressure-area $(\pi-A)$ isotherms after $2 \mathrm{~h}$ and $8 \mathrm{~h}$ incubation for single component $\lambda$-DNA are shown in Fig. 5a. When the incubation time is longer (i.e. $8 \mathrm{~h}$ ), the surface pressure arises from larger surface area and reaches up to higher value of $36 \mathrm{mN} \mathrm{m}^{-1}$ when compared to shorter (i.e. $2 \mathrm{~h}$ ) subphase incubation time. Fig. $5 \mathrm{~b}$ represents normalized isotherms in unit of surface area at the surface pressure of 10 $\mathrm{mN} \mathrm{m}^{-1}$. The normalized isotherm after $8 \mathrm{~h}$ incubation indicates much larger tail around critical surface area than that of $2 \mathrm{~h}$ incubation. These tails indicate the presence of longdistance interaction before phase transition from gas to liquid states suggesting the formation of self-assembled loose and softs of $\lambda$-DNA with increasing incubation time. In fact, when DNA solution spread onto aqueous subphase, initially all the DNA molecules were in random direction in the bulk solution phase. However, with the passage of time before barrier compression, DNA molecules starts to self-assemble together due to weak intermolecular interactions and finally they come at the interface to form their association, resulting the increase of surface pressure. Despite the difference of line shape, the slopes around $10 \mathrm{mN} \mathrm{m}^{-1}$ of surface pressure show the same value for incubation of 2 and $8 \mathrm{~h}$. These results depict the behaviour of $\lambda$-DNA at air-water interface where self-assembling of $\lambda$-DNA molecules proceeds as increasing incubation time but the rigidity of liquid phase is not affected by incubation time.

The incubation effect on single component Cyt $c$ molecules was found to be different from that of $\lambda$-DNA. Fig. $6 a$ and $b$ show the $\pi-A$ isotherms of single component Cyt $c$ with different incubation time, namely 1, 2, 3, 6, 7 and $8 \mathrm{~h}$ after spreading the
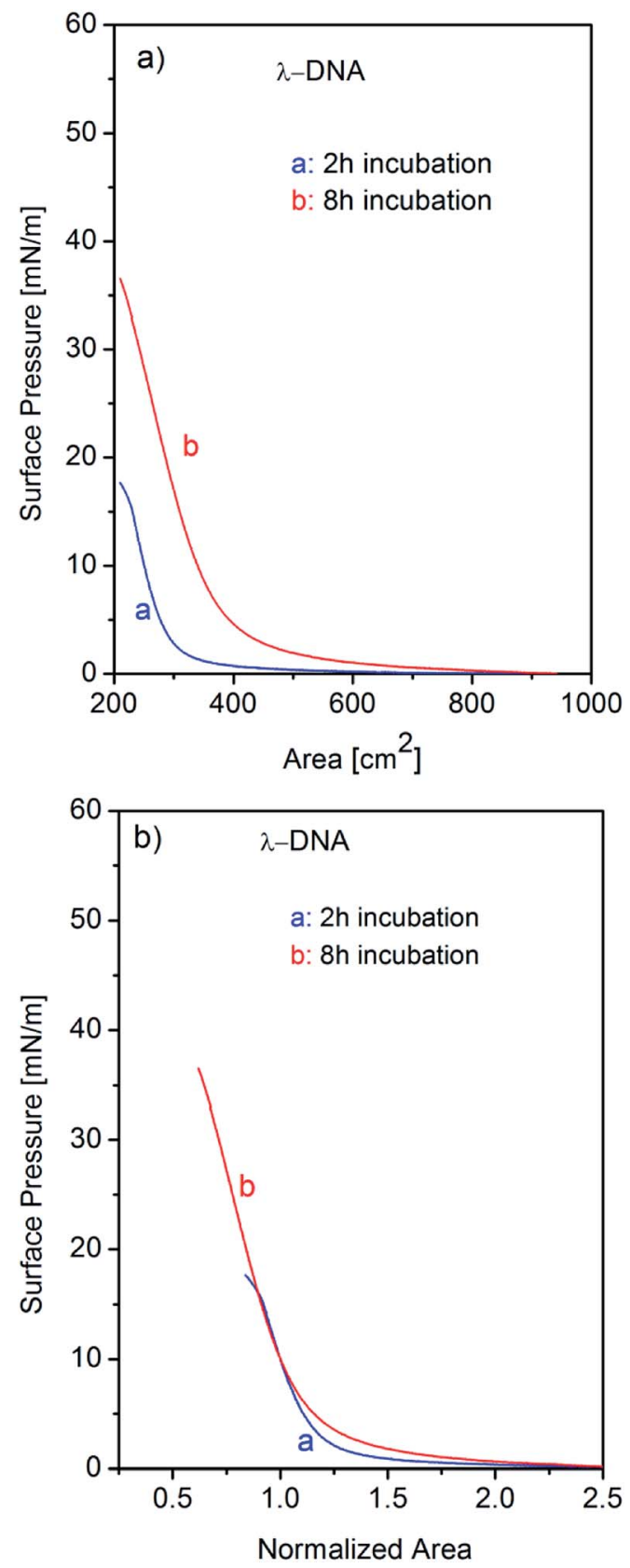

Fig. 5 (a) Surface pressure $(\pi)$ vs. trough area $(A)$ isotherms of single component $\lambda$-DNA at air-water interface recorded after subphase incubation time of $2 \mathrm{~h}$ and $8 \mathrm{~h}$. (b) Corresponding surface pressurearea normalized isotherms of $\lambda$-DNA at a surface pressure of $10 \mathrm{mN}$ $\mathrm{m}^{-1}$. Slope of isotherm does not depend on incubation time.

aqueous solution of Cyt $c$ in the trough and the corresponding surface area-normalized isotherms at the surface pressure of 10 $\mathrm{mN} \mathrm{m}^{-1}$ respectively. The isotherms in Fig. 6a show considerable change as increasing incubation time. As an indication for the number of molecules at air-water interface, the critical surface area can be defined from where there is rapid rise in surface pressure corresponding to a transition from gas to liquid phase of the monolayer. This can be estimated by extrapolating the linear portion of the curve to the area axis. As increasing the 

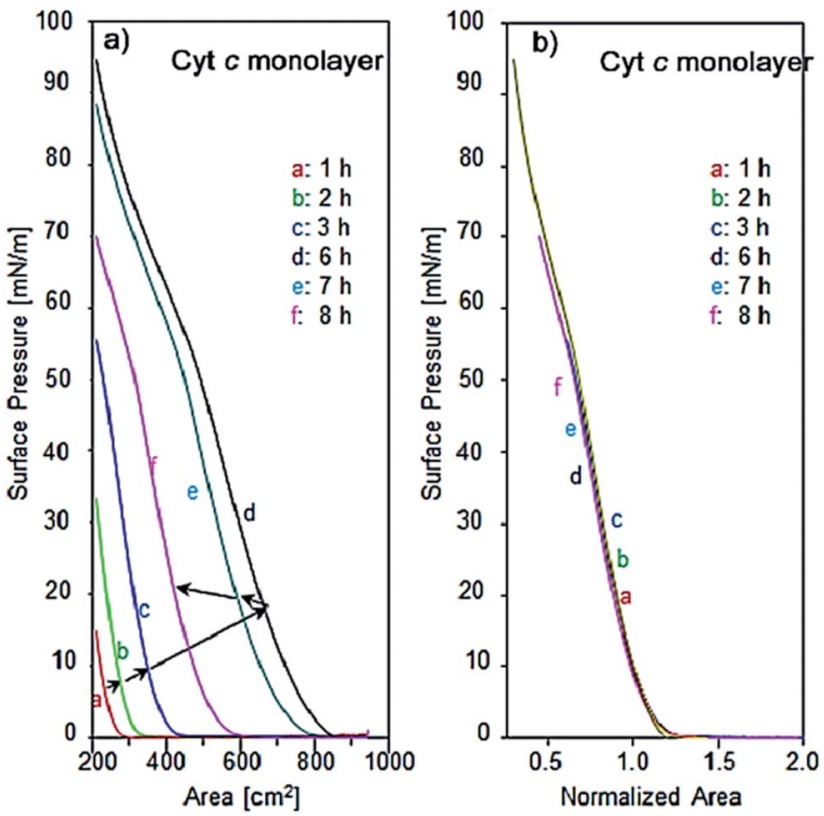

Fig. 6 (a) Surface pressure vs. trough area isotherms of single component Cyt $c$ at the air-water interface recorded after different subphase incubation time viz. 1, 2, 3, 6, 7, 8 h. (b) Corresponding area normalized isotherms of Cyt $c$ at the surface pressure of $10 \mathrm{mN} \mathrm{m}^{-1}$. Shapes of normalized isotherms do not depend on the incubation time, suggesting no influence to monolayer characteristics such as phase transition and the partial aggregation. The effect of prolonged incubation is only the change of the number of molecules.

incubation time up to $6 \mathrm{~h}$, the surface pressure increases three times that of $1 \mathrm{~h}$ incubation, and then a turn for decrease with further incubation. The change in its thermodynamic environment affects stabilities of intermolecular hydrogen bonding, hydrophobic and electrostatic interactions. ${ }^{23}$

In spite of noticeable change of the number of molecules at air-water interface, the normalized isotherms as shown in Fig. $6 \mathrm{~b}$ are overlapped completely as the same curve suggesting that the long-time incubation affects merely the number of Cyt $c$ molecules determined by diffusion and equilibrium between the bulk and the interface. It suggests no influence to monolayer characteristics such as phase transition and partial aggregation. All the normalized isotherms of Cyt $c$ show the reproducible shoulder at the surface pressure of $52 \mathrm{mN} \mathrm{m}^{-1}$ where the slope of isotherms become slow upon compression. The presence of this shoulder implies that the protein is more susceptible to conformational changes at the air-water interface followed by more cohesive interactions in Langmuir monolayer. ${ }^{35}$ This is possibly due to the change in orientation of the heme group of Cyt $c$ upon compression of the monolayer or may be redissolving of the protein in the trough. ${ }^{36}$

In the present work, we study the formation of Cyt $c / \lambda$-DNA complex molecular assemblies at air-water interface for various incubation times after spreading the mixed solution of $\lambda$-DNA and Cyt $c$ onto the trough before the barrier compression. The isotherms of Cyt $c / \lambda$-DNA mixed system show characteristic behaviours which are different from both single component system of Cyt $c$ and $\lambda$-DNA. Fig. 7a shows the surface pressure $v s$.
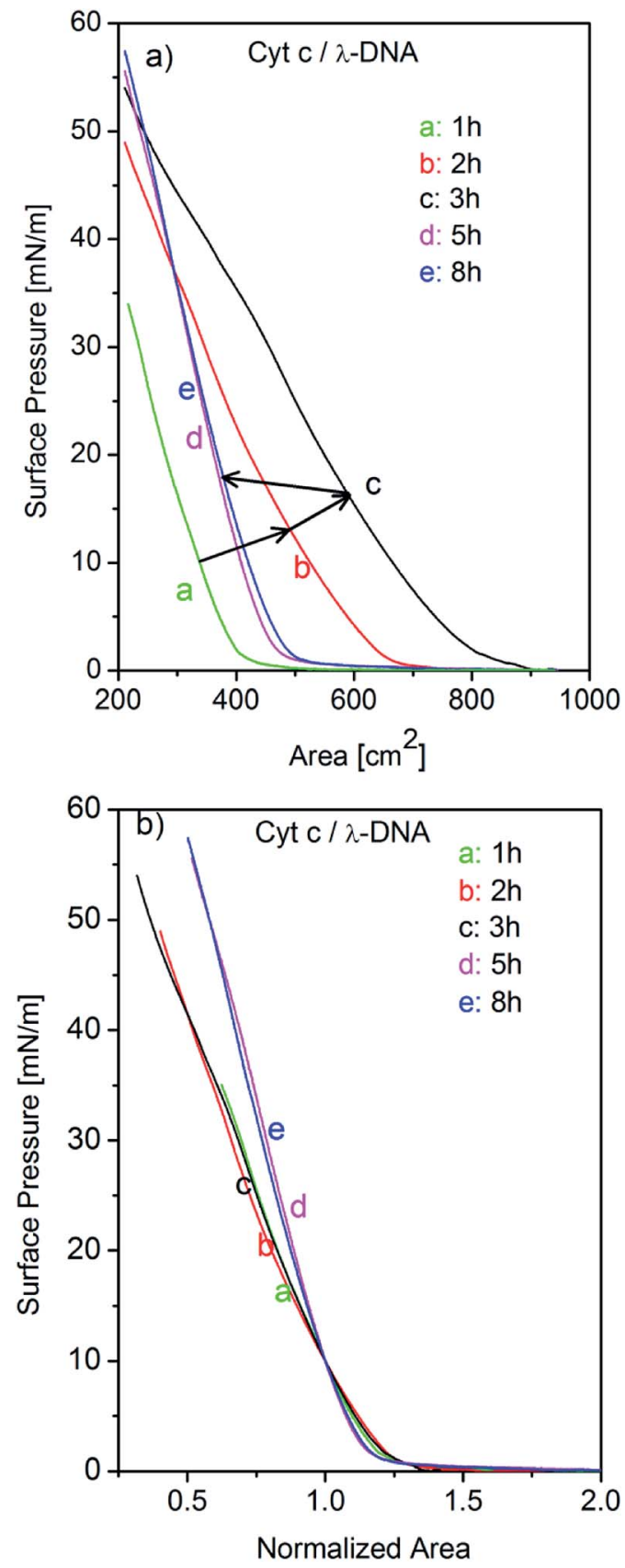

Fig. 7 (a) Surface pressure vs. trough area isotherms of Cyt $c / \lambda$-DNA complex molecular assemblies at air-water interface recorded after different subphase incubation time viz. 1, 2, 3, 5, 8 h. (b) Corresponding area-normalized isotherms of complex Cyt $c / \lambda$-DNA assemblies at the surface pressure of $10 \mathrm{mN} \mathrm{m}{ }^{-1}$. Slope shows two categories: one is similar to single component $\lambda$-DNA before $3 \mathrm{~h}$ incubation, and the other is similar to that of Cyt $c$ alone after $5 \mathrm{~h}$.

area $(\pi-A)$ isotherms of Cyt $c / \lambda$-DNA mixed monolayer for different incubation times of $1,2,3,5$ and $8 \mathrm{~h}$ at the air-water interface. From the isotherms it is observed that surface area of the Cyt $c / \lambda$-DNA complex monolayer increases with increase in incubation time and reaches maximum for incubation within $3 \mathrm{~h}$. On further increase in incubation time, the surface pressure decreases. The plots of critical surface area for the isotherms of 
Cyt $c / \lambda$-DNA along with that of single component Cyt $c$ monolayer are shown in Fig. 8. We immediately observe that initially (up to $3 \mathrm{~h}$ ) the critical area of Cyt $c / \lambda$-DNA complex monolayer goes up and the area value is much greater than that of single component Cyt $c$ monolayer. Interestingly, after $3 \mathrm{~h}$ of incubation, the area of Cyt $c / \lambda$-DNA complex monolayer at a particular surface pressure is somewhat less than that of single component Cyt $c$ monolayer at air-water interface.

The normalized isotherms of Cyt $c / \lambda$-DNA complex was found to be different from that of single component Cyt $c$ as shown in Fig. 7b. The slopes show constant value until $3 \mathrm{~h}$ of incubation and then become steep with fixed value after $3 \mathrm{~h}$ of incubation. The correspondence of this discontinuity of the slopes in normalized isotherms with the saturation of critical surface area mentioned above at subphase incubation time of $3 \mathrm{~h}$ suggests following behaviours of Cyt $c / \lambda$-DNA complexes. This rapid increase of critical surface area of Cyt $c / \lambda$-DNA complex monolayer at air-water interface is shown in Fig. 8. It is only due to the increase in number of complex molecules at the interface because the overall shapes of normalized isotherms do not change during this increase. The Cyt $c$ and $\lambda$-DNA molecules in bulk solution are incorporated into insoluble complex molecular network and the extent of the formation of this assembly increases with the passage of time and is in consistent with the result of the AFM observation as shown in Fig. 4.

However, after $3 \mathrm{~h}$ of incubation, the rate of adsorption decreases because of the formation of larger Cyt $c / \lambda$-DNA aggregates which is also confirmed by AFM measurements. Cyt $c / \lambda$-DNA complex Langmuir monolayer at surface pressure of 10 $\mathrm{mN} \mathrm{m}{ }^{-1}$ after incubating the monolayer subphase for $5 \mathrm{~h}$ was deposited on a mica substrate by horizontal dipping method

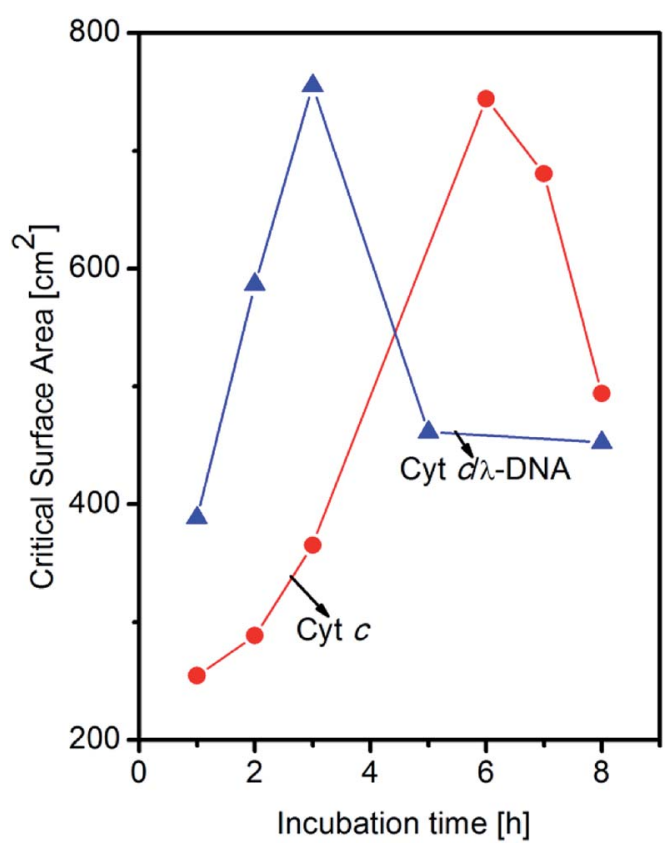

Fig. 8 Change in critical surface area as a function of different incubation time before barrier compression at air-water interface for single component Cyt $c$ and Cyt $c / \lambda$-DNA complex. For Cyt $c / \lambda$-DNA complex, rapid rise and drop are observed. and the morphology of the film was observed by AFM. Fig. 9 shows the AFM topographic image of Cyt $c / \lambda$-DNA complex LB film after $5 \mathrm{~h}$ of subphase incubation. From this image we immediately recognize very large complex molecular aggregates some of them are having rod-like structures. In fact for such prolonged subphase incubation more number of Cyt $c / \lambda$-DNA complex come at the interface. The strong columbic attractive interaction between Cyt $c$ and DNA also results self-aggregation $^{37}$ of Cyt $c$ in the backbone of DNA chains at air-water interface. The section profile shows the height of these aggregates in the range from 4 to $8 \mathrm{~nm}$. In comparison with the AFM image of complex LB film deposited after $2 \mathrm{~h}$ incubation (see Fig. 4), the surface morphology is changed completely with long time incubation. Also during surface compression by barriers larger aggregates are formed due to more closer association of DNA chains having Cyt $c$ bound in their backbone and these larger sized Cyt $c / \lambda$-DNA complex aggregates eventually precipitate faster as bulk in the subphase with the passage of time resulting rapid decrease in theoretical surface area of Cyt $c / \lambda$ DNA complex monolayer (Fig. 8). As a consequence the Cyt $c / \lambda$ DNA complex molecular network became fragmented at airwater interface. Therefore, the rod-like morphology of complex LB film when transferred onto mica after such a prolonged subphase incubation time is possibly due to aggregated rigid and fragmented complex molecular distribution instead of a continuous network. Such type of aggregation is not observed in single component of each Cyt $c$ and $\lambda$-DNA monolayer.
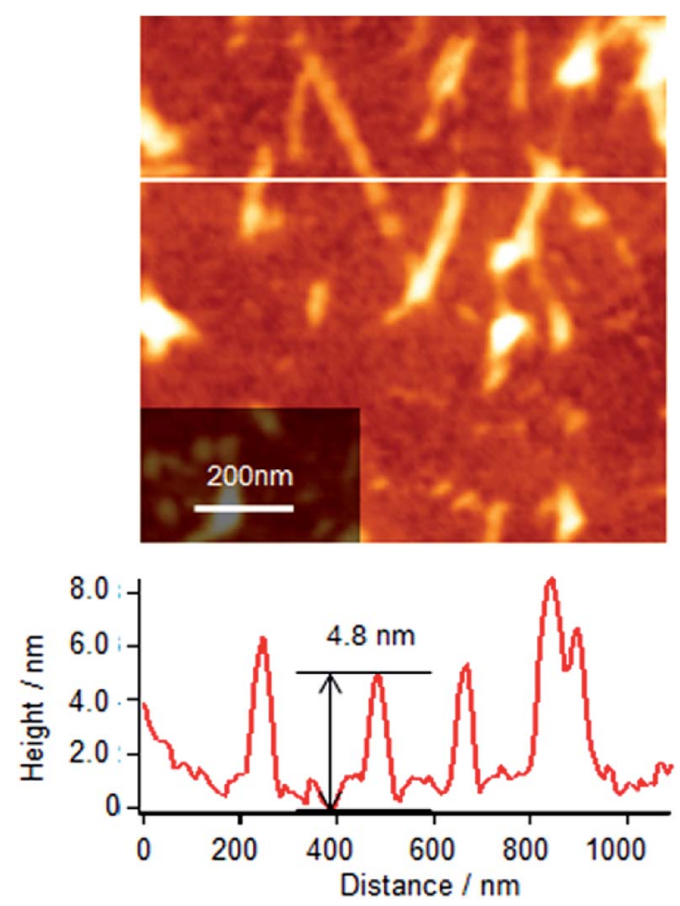

Fig. 9 AFM topographic image of Cyt $c / \lambda$-DNA complex Langmuir monolayer transferred onto mica substrate at the surface pressure of $10 \mathrm{mN} \mathrm{m}^{-1}$ by horizontal dipping method. Barrier compression was done after $5 \mathrm{~h}$ of spreading Cyt $c / \lambda$-DNA mixed solution. Rod-like large aggregates are observed. The section profile for the aggregates gives the height in the range from 4 to $8 \mathrm{~nm}$. 


\subsection{Rigidity of interfacial monolayers: two-dimensional Young's modulus}

The comparable consideration concerning normalized isotherms for three different systems, namely single component $\lambda$-DNA, single component Cyt $c$, and Cyt $c / \lambda$-DNA complex, is useful to understand three different interactions which are probably responsible for the formation of Langmuir monolayers: (1) DNA-DNA interaction, (2) DNA-protein interaction and (3) protein-protein interaction. As mentioned above, the slopes of normalized isotherms correspond to two dimensional Young's modulus. The values of the two-dimensional Young's modulus are summarized in Table 1 . The values for $\lambda$-DNA and Cyt $c / \lambda$-DNA complex monolayer before $3 \mathrm{~h}$ incubation are very similar to each other. This is because both the isotherms reflect intrinsically the compression of DNA networks formed at airwater interface. As a result, the slopes for these systems represent almost the same Young's modulus around $50 \mathrm{mN} \mathrm{m}^{-1}$ for two-dimensional DNA network. However, on the passage of compression, the surface pressure of $\lambda$-DNA arises gradually, while that of Cyt $c / \lambda$-DNA complex shows clear threshold. This difference might come from the difference of molecular interaction involved, namely, long-chained $\lambda$-DNA molecules give some surface pressure from the weak and loose self-assembled network formation before reaching to critical surface area. In the meantime, the interaction between cationic Cyt $c$ and anionic $\lambda$-DNA molecules generates rigid and restrictive fragments at the air-water interface resulting no surface pressure before reaching critical surface area. Schematic illustration of the monolayer compression for single component Cyt $c$, single component $\lambda$-DNA, and Cyt $c / \lambda$-DNA complex at air-water interface is shown in Fig. 10.

In contrast, Young's modulus of single component Cyt $c$ monolayer shows the large value of $96 \mathrm{mN} \mathrm{m}^{-1}$. This means that Cyt $c$ molecule behaves like a hard sphere and forms cohesive monolayer. The value is also almost the same for the normalized isotherms of Cyt $c / \lambda$-DNA complex assemblies after $3 \mathrm{~h}$ incubation. This also implies that the large aggregates of Cyt $c /$ $\lambda$-DNA complex is not network but hard body particles or fragmented rigid rod-like aggregates in consistent with the observed AFM image as shown in Fig. 9.

As mentioned earlier that this $\alpha$-helical redox protein Cyt $c$ has the isoelectric point (pI) about $10-10.5 .^{27}$ So, in this work, the positively charged lysin residues of Cyt $c$ interacted with negatively charged phosphate backbone of $\lambda$-DNA forming Cyt

Table 1 Two-dimensional Young's modulus of single component $\lambda$ DNA, single component Cyt $c$ and Cyt $c / \lambda$-DNA complex interface monolayers obtained from surface-area-normalized isotherms at surface pressure of $10 \mathrm{mN} \mathrm{m}^{-1}$

\begin{tabular}{|c|c|c|c|c|}
\hline \multirow[b]{2}{*}{ System } & \multirow{2}{*}{$\begin{array}{l}\lambda \text {-DNA } \\
\left(\mathrm{mN} \mathrm{m}^{-1}\right) \\
\text { monolayer }\end{array}$} & \multirow{2}{*}{$\begin{array}{l}\text { Cyt } c \\
\left(\mathrm{mN} \mathrm{m}^{-1}\right) \\
\text { monolayer }\end{array}$} & \multicolumn{2}{|c|}{$\begin{array}{l}\text { Cyt } c / \lambda \text {-DNA monolayer } \\
\left(\mathrm{mN} \mathrm{m}^{-1}\right)\end{array}$} \\
\hline & & & Before $3 \mathrm{~h}$ & After $3 \mathrm{~h}$ \\
\hline $\begin{array}{l}\text { Young's } \\
\text { modulus }\end{array}$ & 49 & 96 & 54 & 96 \\
\hline
\end{tabular}

$c / \lambda$-DNA molecular complex in aqueous solution and thereafter organized at air-water interface. However, the extent of interaction may depend upon different microenvironment. ${ }^{32}$ For the complex monolayer at the interface, DNA has been used as the building block for the Cyt $c / \lambda$-DNA complex molecular network. In our work, $\mathrm{pH}$ of the subphase after spreading Cyt $c / \lambda$-DNA mixed solution was 6.2. It is already reported elsewhere ${ }^{32}$ that acidic condition is more favourable for Cyt $c / \lambda$-DNA interaction. So, in the present experiment initially when Cyt $c / \lambda$-DNA mixed solution was spread onto subphase, the DNA chains having Cyt $c$ adsorbed onto its surface start to self-associate at the air-water interface with the passage of time. This leads to the increase in monolayer area coverage as well as surface pressure.

\subsection{Polarization of Cyt $c / \lambda$-DNA complex LB film probed by electrostatic force microscopy}

To confirm the presence of ionic interaction between Cyt $c$ and $\lambda$-DNA, simultaneous observation of AFM topography and electrostatic force microscopy (EFM) images was performed as shown in Fig. 11 for Cyt $c / \lambda$-DNA complex LB monolayer transferred onto mica by horizontal dipping method at a surface pressure of $10 \mathrm{mN} \mathrm{m}^{-1}$ after $2 \mathrm{~h}$ incubation. In the topography image (Fig. 11a), the bright particle like protrusions are observed on DNA chain. These protrusions are 1-2 nm corresponding to the height of Cyt $c$ molecules that slightly compressed by adsorption on a surface. On the basis of this identification, EFM image as shown in Fig. 11b represents one-
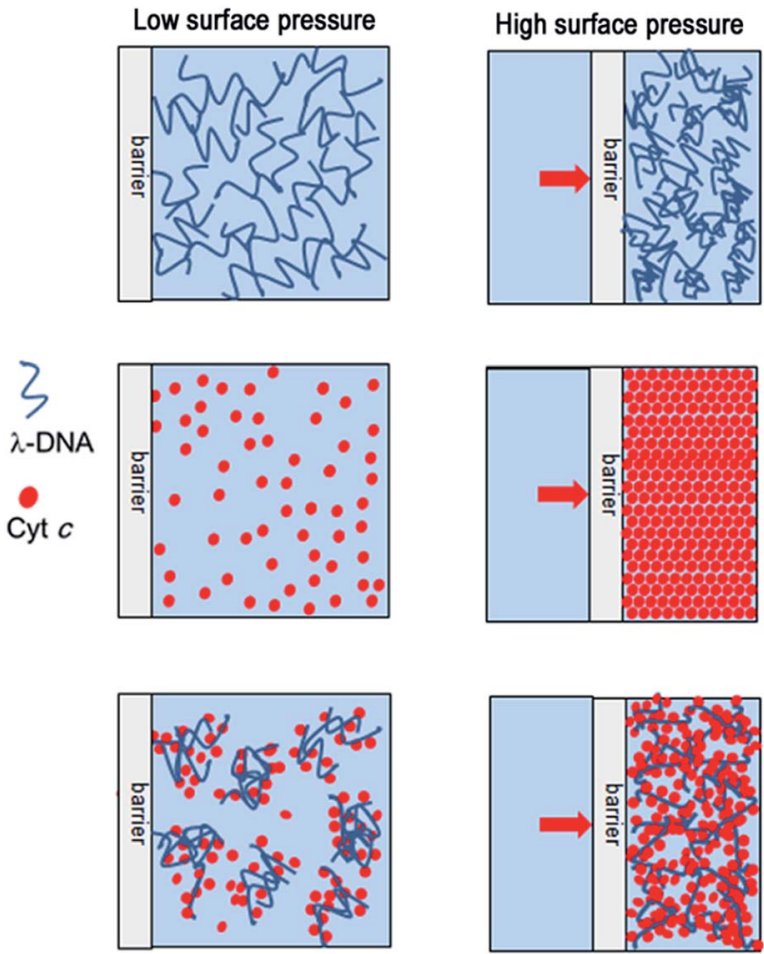

Fig. 10 Schematic illustration for the molecular assemblies of single component $\lambda$-DNA, single component $C y t c$ and $C y t c / \lambda$-DNA complex at the air-water interface in the low and high surface pressure region upon barrier compression. 
a)

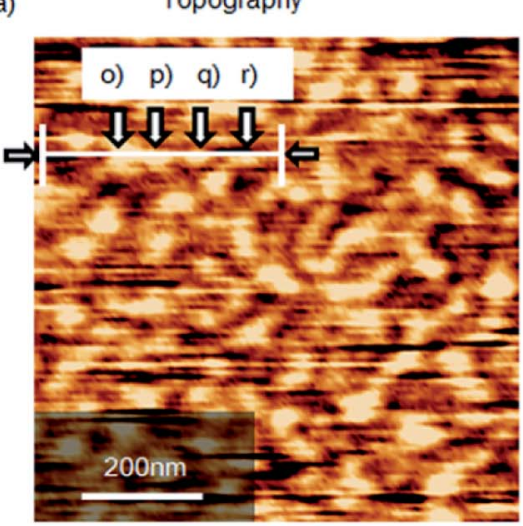

o) p)

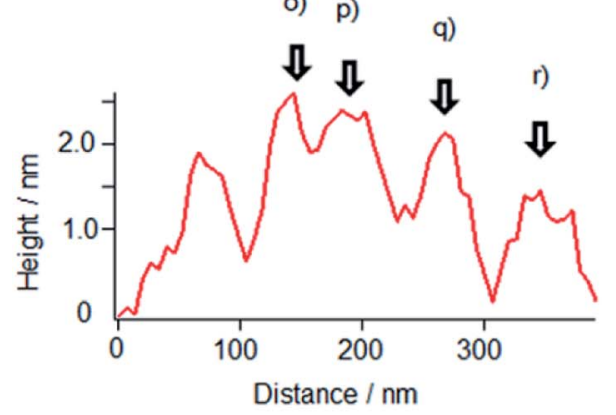

b)

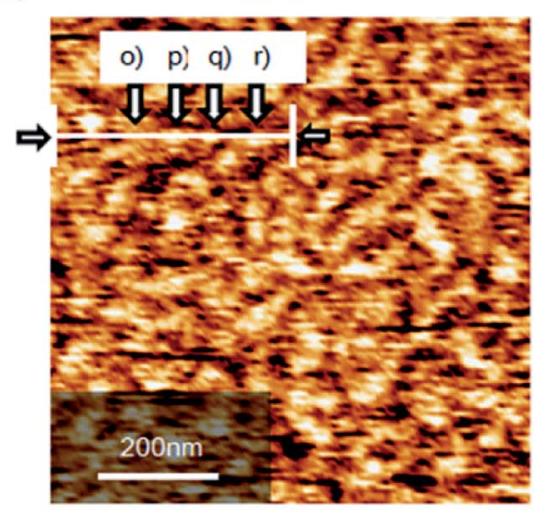

p) q)

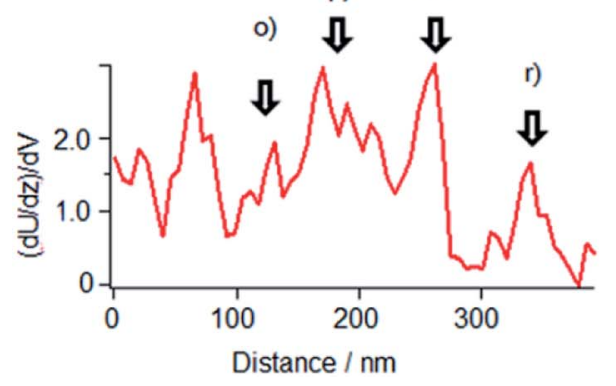

Fig. 11 Frequency mode non-contact (a) AFM topographic image and (b) simultaneous electrostatic force microscopy (EFM) image of Cyt $\mathrm{Cl}$ $\lambda$-DNA complex LB film deposited onto mica at the surface pressure of $10 \mathrm{mN} \mathrm{m}^{-1}$. Barrier compression was done after $2 \mathrm{~h}$ of spreading Cyt $\mathrm{cl}$ $\lambda$-DNA mixed solution onto the subphase.

by-one correspondence between topographical structure and electrical polarization in molecular level. Generally, it is well known that DNA on a mica surface has negative charge with regard to mica substrate. So, the bright contrast of $\lambda$-DNA molecules in EFM image means that the positive end of the dipole between $\lambda$-DNA molecule and mica surface face to the substrate. Similarly, the EFM image of the most part of Cyt $c$ molecules has bright contrast indicating dipole along the same direction. This result implies that Cyt $c$ molecules adsorb on not a mica surface but DNA molecules because oppositely directed dipole should be generated by the direct adsorption of positively charged Cyt $c$ molecules to a mica surface. As mentioned above, the most part of Cyt $c$ molecules give bright contrast but small number of the molecules shows wide variation as shown in section profiles. The protrusions in topography image denoted by (p), (q), (r) correspond to the bright EFM contrasts of (p), (q), (r), respectively but in case of (o), EFM contrast is almost transparent. These results definitely suggested that Cyt $c$ molecules are bonded to $\lambda$-DNA and Cyt $c / \lambda$-DNA complexes have diversity of the structure and orientation.

\section{Conclusions}

This work demonstrates that two highly water soluble biomaterials namely $\lambda$-DNA and a redox protein Cyt $c$ can make complex Langmuir interfacial layer at air-water interface. Surface pressure of single component systems of $\lambda$-DNA or Cyt $c$ and their mixtures were increased gradually upon barrier compression after spreading their solution in the trough. We have succeeded to separate the two effects namely the phase change and the number of molecules involved in monolayer formation at air-water interface with the passage of time by considering normalized isotherms and critical surface area. Two-dimensional Young's modulus essentially compares the rigidity of the monolayers of single component $\lambda$-DNA, single component Cyt $c$ and Cyt $c / \lambda$-DNA complex thereby confirming the formation of their complex molecular architecture at airwater interface after various incubation times. The molecular level interactions in the complex monolayer were found to be same as that of single component $\lambda$-DNA during the first few hours of subphase incubation. However, it shows similar tendency to that of single component Cyt $c$ monolayer at airwater interface. The decrease in surface pressure of Cyt $c / \lambda$-DNA complex network at larger incubation time was due to the formation of rod-like aggregates as was also evidenced by AFM image of complex LB film deposited onto mica after $5 \mathrm{~h}$ of subphase incubation.

\section{Acknowledgements}

This work is supported by a Grant-in-Aid for Scientific Research on Innovative Areas "Molecular Architectonics: Orchestration of Single Molecules for Novel Functions" (25110014), Scientific Research (B) (24360011) from the Ministry of Education, Culture, Sports, Science and Technology of Japan (MEXT). P. K. Paul is greatly thankful to Osaka University for giving financial assistance (Osaka University Visiting Scholar Program, 13IS013) to perform the work. 


\section{References}

1 C. G. Kalodimos, N. Biris, A. Bonvin, M. Levandoski, M. Guennuegues, R. Boelens and R. Kaptein, Science, 2004, 305, 386.

2 O. Littlefield and H. C. M. Nelson, Protein. Struct. Funct. Genet., 2001, 45, 219.

3 R. P. Goodman, R. M. Berry and A. J. Turberfield, Chem. Commun., 2004, 1372.

4 S. Liao and N. C. Seeman, Science, 2004, 306, 2072.

5 G. Liu, Y. Wan, V. Gau, J. Zhang, L. Wang, S. Song and C. Fan, J. Am. Chem. Soc., 2008, 130, 6820.

6 Z. Peng and H. Liu, Chem. Mater., 2016, 28, 1012.

7 J. Deisenhofer, O. Epp, K. Miki, R. Huber and H. Michel, Nature, 1985, 318, 618.

8 A. Langer, P. A. Hampel, W. Kaiser, J. Knezevic, T. Welte, V. Villa, M. Maruyama, M. Svejda, S. Jähner, F. Fischer, R. Strasser and U. Rant, Nat. Commun., 2013, 2099, DOI: 10.1038/ncomms3099.

9 C. M. Niemeyer, Angew. Chem., Int. Ed., 2010, 49, 1200.

10 D. H. Coombs and G. D. Pearson, Proc. Natl. Acad. Sci. U. S. A., 1978, 75, 5291.

11 A. Kazuya and M. Komiyama, Nanoscale, 2010, 2, 309.

12 J. D. Flory, C. R. Simmons, S. Lin, T. Johnson, A. Andreoni, J. Zook, G. Ghirlanda, Y. Liu, H. Yan and P. Fromme, J. Am. Chem. Soc., 2014, 136, 8283.

13 F. F. Andersen, B. Knudsen, C. L. P. Oliveira, R. F. Frohlich, D. Kruger, J. Bungert, M. Agbandje-McKenna, R. McKenna, S. Juul, C. Veigaard, J. Koch, J. L. Rubinstein, B. Guldbrandtsen, M. S. Hede, G. Karlsson, A. H. Andersen, J. S. Pedersen and B. R. Knudsen, Nucleic Acids Res., 2008, 36, 1113.

14 C. M. Erben, R. P. Goodman and A. J. Turberfield, Angew. Chem., Int. Ed., 2006, 45, 7414.

15 H. Yamaguchi, D. C. Che, Y. Hirano, M. Suzuki, Y. Higuchi and T. Matsumoto, Jpn. J. Appl. Phys., 2015, 54, 095201.

16 T. G. Drummond, M. G. Hill and J. K. Barton, Nat. Biotechnol., 2003, 21, 1192.

17 Y. Hirano, Y. Segawa, T. Kawai and T. Matsumoto, J. Phys. Chem. C, 2013, 117, 140.

18 D. Sarauli, J. Tanne, D. Schaefer, I. W. Schubart and F. Lisdat, Electrochem. Commun., 2009, 11, 2288.
19 T. Kanno, H. Tanaka, N. Miyoshi and T. Kawai, Jpn. J. Appl. Phys., 2000, 39, L269.

20 S.-I. Tanaka, Y. Maeda, L.-T. Cai, H. Tabata and T. Kawai,Jpn. J. Appl. Phys., 2001, 40, 4217.

21 A. S. Haas, D. L. Pilloud, K. S. Reddy, G. T. Babcock, C. C. Moser, J. K. Blasie and P. L. Dutton, J. Phys. Chem. B, 2001, 105, 11351.

22 C. Hansda, S. A. Hussain, D. Bhattacharjee and P. K. Paul, Surf. Sci., 2013, 617, 124.

23 L. Razumovsky and S. Damodaran, Langmuir, 1999, 15, 1392.

24 X. Chen, J. Wang, N. Shen, Y. Luo, L. Li, M. Liu and R. K. Thomas, Langmuir, 2002, 18, 6222.

25 X. Dai, C. Wei, Z. Li, Z. Sun, R. Shen and Y. Zhang, RSC Adv., 2013, 3, 16116.

26 M. Hüttemann, S. Helling, T. H. Sanderson, C. Sinkler, L. Samavati, G. Mahapatra, A. Varughese, G. Lu, J. Liu, R. Ramzan, S. Vogt, L. I. Grossman, J. W. Doan, K. Marcus and I. Lee, Biochim. Biophys. Acta, Bioenerg., 2012, 1817, 598.

27 G. W. Bushnell, G. V. Louie and G. D. Brayer, J. Mol. Biol., 1990, 214, 585.

28 A. N. Volkov and N. A. J. van Nuland, PLoS Comput. Biol., 2012, 8, e1002807.

29 P. B. Crowley, E. Chow and T. Papkovskaia, ChemBioChem, 2011, 12, 1043.

30 M. P. Pileni, T. Zemb and C. Petit, Chem. Phys. Lett., 1985, 118, 414.

31 P. Brochette, C. Petit and M. P. Pileni, J. Phys. Chem., 1988, 92, 3505.

32 C. Wettstein, C. Kyne, A. M. Doolan, H. Möhwald, P. B. Crowley and F. Lisdat, Nanoscale, 2014, 6, 13779.

33 H. J. Bolink, E. Baranoff, M. Clemente-Leon, E. Coronado, N. Lardies, A. Lopez-Munoz, D. Repetto and M. K. Nazeeruddin, Langmuir, 2008, 26, 11461.

34 E. Mikamo-Satoh, F. Yamada, A. Takagi, T. Matsumoto and T. Kawai, Nanotechnology, 2009, 20, 145102.

35 K. Sankaranarayanan, B. U. Nair and A. Dhathathreyan, Appl. Surf. Sci., 2013, 273, 75.

36 M. Saint-Pierre-Chazalet, F. Billoudet and M. P. Pileni, Prog. Colloid Polym. Sci., 1989, 79, 76.

37 X. Jiang, Y. Wang, X. Qu and S. Dong, Biosens. Bioelectron., 2006, 22, 49. 